\title{
Peculiar properties of the electron beam dynamics simulation by particle-particle methods taking into account delay effects
}

\author{
Sergey Alikov ${ }^{1, *}$ and Alexandr Shein ${ }^{1}$ \\ ${ }^{1}$ Volgograd State Technical University, Physics Department, 400131, Volgograd city, \\ Russian Federation
}

\begin{abstract}
An analysis of the plane influence separating the interaction space from the electron beam-forming region is made by the particleparticle simulation method with the delay effect. During the simulation, it was shown that the boundary conditions on the surface have a significant effect on the electron beam dynamics in a longitudinal magnetic field and affect the time of formation of the virtual cathode and the particle velocity in the interaction space.
\end{abstract}

\section{Particle-particle method with the delay effect}

The development of effective algorithms and methods for electron beams simulation is one of the important objectives of physical electronics. Recently, particle-in-cell methods have become widespread [1-3]. But the implementation of these methods is based on the charge deposition between the mesh nodes by the weighting scheme. The choice of weighting schemes for the space charge leads to the appearance of mesh noises and mesh effects. For this reason, in [4], a particle-particle method for electron beams simulation taking into account the delay effect was proposed. This method has proved itself in studies of beams in crossed electric and magnetic fields [5]; it was used to study beams over an ideal conducting plane [6], and in neutral gas [7].

One of the boundary surfaces that substantially affects the beam is the surface of the grid (or cathode) through which it enters the interaction space. The influence of the grid on the development of virtual cathode for the beam in the uniform magnetic field in the particle-particle approach is the main part of this work. The second goal of the authors was to show some of the calculation algorithm features and implementation details.

\subsection{Motion equations and space charge field}

The numerical model is based on the method of super-particles. The beam is represented as a combination of super-particles which masses and charges are $\mathrm{k}$ times greater than the mass and charge of the electron. Next, the equations of motion are solved:

*Corresponding author: sputnik_as@mail.ru 


$$
\frac{d \mathbf{p}_{i}}{d t}=q_{i}\left[\mathbf{E}_{e x}\left(\mathbf{r}_{i}, t\right)+\sum_{j} \mathbf{E}_{j i}+\mathbf{v}\left(\mathbf{p}_{i}\right) \times\left(\mathbf{B}_{e x}\left(\mathbf{r}_{i}, t\right)+\sum_{j} \mathbf{B}_{j i}\right)\right], \quad \frac{d \mathbf{r}_{i}}{d t}=\mathbf{v}\left(\mathbf{p}_{i}\right) .
$$

Here $\mathbf{E}_{e x}, \mathbf{B}_{e x}$ are the external electric and magnetic field, $\mathbf{v}\left(\mathbf{p}_{i}\right)$ is the particle velocity, $\mathbf{p}_{i}$ is its momentum, $q_{i}$ is the charge of super-particle, $m_{i}$ is its mass. $\mathbf{E}_{j i}, \mathbf{B}_{j i}$ are the electric and magnetic field created by the $j$-th particle at the point with the $\mathrm{i}$-th particle. They are determined by the formulas of the Lienard-Wiechert theory:

$$
\begin{gathered}
\mathbf{E}_{j i}=\left.\frac{q_{j}}{4 \pi \varepsilon_{0}} \frac{1}{R^{2}\left(1-\frac{\mathbf{n} \cdot \mathbf{v}_{j}}{c}\right)^{3}}\left\{\left(\mathbf{n}-\frac{\mathbf{v}_{j}}{c}\right)\left(1-\frac{\mathbf{v}_{j}^{2}}{c^{2}}+\frac{R}{c^{2}} \mathbf{n} \cdot \mathbf{a}_{j}\right)-\frac{R}{c^{2}} \mathbf{a}_{j}\left(1-\frac{\mathbf{n} \cdot \mathbf{v}_{j}}{c}\right)\right\}\right|_{\tau}, \\
\mathbf{B}_{j i}=\left.\frac{\mathbf{n} \times \mathbf{E}_{j i}}{c}\right|_{\tau} .
\end{gathered}
$$

Here $\mathbf{n}=\mathbf{R} / R, \mathbf{R}=\mathbf{r}_{i}(t)-\mathbf{r}_{j}(\tau), R=|\mathbf{R}|$. The moment of time in the past $\tau$ is determined from the solution of the equation:

$$
c(t-\tau)=R
$$

\subsection{Peculiar properties of the numerical implementation and the search for the moment in time in the past}

The equations of motion were solved by the fourth-order Runge-Kutta method in the numerical implementation, the method time-step was fixed, we denote it by $\Delta \mathrm{t}$. The enlargement coefficient was calculated from the known value of the injection area $\mathrm{S}$, the number of particles entering the interaction space in step $\mathrm{N}$ and current $\mathrm{I}$, or, if necessary, from the known space charge density $\rho$ and the initial electron velocity $v$, which was assumed to be the same for all particles:

$$
k=\frac{|I| \Delta t}{|e| N}=\frac{|\rho| v S \Delta t}{|e| N} .
$$

Particles crossed the ejection site with a uniform distribution over the area. The beamforming system was not considered, and it was assumed that the flow is uniform in volume in front of the ejection site. For this reason, the initial coordinates along the $z$ axis (Fig. 1) were evenly distributed from $\mathrm{z}=0$ to $\mathrm{z}=\mathrm{v} \Delta \mathrm{t}$.

The main computational complexity of the problem is to calculate the space-charge field. If we denote the number of time steps at the moment $\mathrm{t}$ by $N_{t}$, then for a problem without the delay effect, it would be $O\left(N^{2} N_{t}^{2}\right)$. For a problem with the delay effect, everything will depend on the method of solving equation (4). If the solution is carried out by the simple descent method by reducing the time by the timestep, until the function $f(\tau)=c(t-\tau)-\left|\mathbf{r}_{i}(t)-\mathbf{r}_{j}(\tau)\right|$ will change the sign, then the complexity will be $O\left(N^{2} N_{t}^{3}\right)$. If you use the bisection method, then it will be $O\left(N^{2} N_{t}^{2} \ln N_{t}\right)$. Another way is to limit the number of steps in the descent method, then the complexity will become as in the method without the delay effect, but it will have low accuracy. 


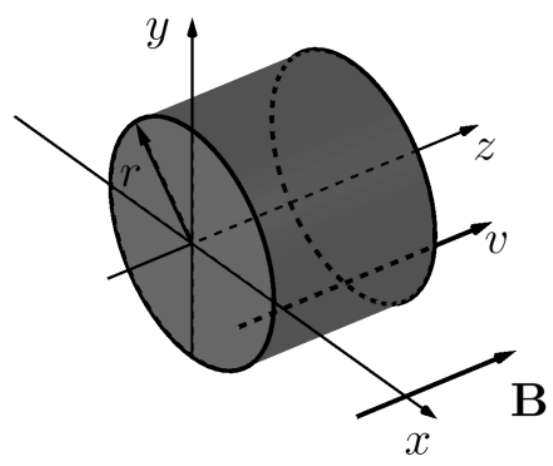

Fig. 1. The geometry of the system: the cylindrical electron beam ejected from the plane $z=0$ is transported in the longitudinal magnetic field $\mathrm{B}$ cross the $\mathrm{z}$ axis.

The most effective solution is the bisection method. At the considered time intervals, it gives results comparable in speed to the descent method with a limited number of steps, but at the same time it does not lead to loss of accuracy. It should also be noted that all the presented methods find only the step in the numerical scheme on which the function $f(\tau)$ changes the sign. So the accuracy of calculations decreases, but this error is not large compared to the fact that the space charge field is not recalculated in the Runge-Kutta method. The latter is associated with the features of storing data in memory and it can be used if the space charge field is significantly weaker than the external electromagnetic field.

The bisection method for equation (4) is shown on the Figure 2. We sequentially construct two spheres that the point at which we are looking for an electromagnetic field is between the spheres. For this reason, it is sometimes called the method of spheres, as well as the method of descent above.

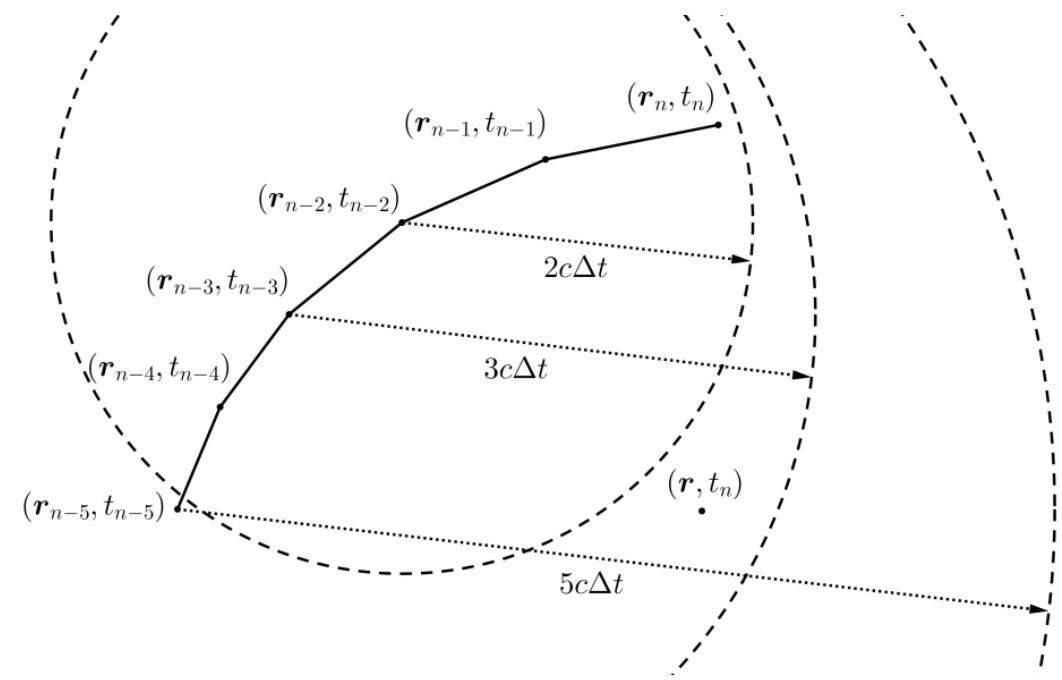

Fig. 2. The bisection method for searching the time moment in the past. First, we construct the first sphere for the maximum moment in the past when the particle appeared in the interaction space - in this case, a sphere of radius $5 \mathrm{c} \Delta \mathrm{t}$ for $\mathrm{n}-5$ step. Then we divide the number 5 by 2 in order to build the next sphere with radius $2 c \Delta t$, for step $n-2$. We find the sphere lying between them for $(5+2) / 2=3$, $\mathrm{n}-3$ step. We continue the process until the radii of the spheres differ by $\mathrm{c} \Delta \mathrm{t}$. In the latter case, the process is terminated. Due to the peculiarities of modeling, in the past, we take the moment in the time corresponding to the sphere with larger radius - in this case, $\mathrm{n}-3$. 
The model was tested on the problems of a particle in crossed fields, on the problem of expanding a uniform ultrarelativistic charged ball. The calculation method showed good agreement between the results and theory. The time step and the number of ejected particles per step were selected based on the condition $\omega_{c} \Delta t, \omega_{p} \Delta t<<1$ and comparing the calculation results with different time steps and the number of particles between them. Here $\omega_{c}=|q| B / m, \quad \omega_{p}=\sqrt{|\rho q| / m \varepsilon_{0}}$, the cyclotron and plasma frequencies, respectively.

\subsection{Method of images for the perfectly conducting injector plane}

Theoretical models of the phenomenon the virtual cathode usually are considered the system that consists of cathode with zero potential, collector or anode, and the conducting grid with accelerating potential [8]. We will only consider the effect of the perfectly conducting grid on the formation of the virtual cathode, removing the collector and side walls of the device from consideration. Assuming that the grid is perfectly conducting, we get that the tangential component of the electric field should be equal to zero on it (Fig. 3). To do this, consider the image of our particle with a charge $-q_{j}$, which moves along the mirror path of the $\mathrm{j}$-th particle $\left(\mathrm{x}_{\mathrm{j}}(\mathrm{t}), \mathrm{y}_{\mathrm{j}}(\mathrm{t}),-\mathrm{z}_{\mathrm{j}}(\mathrm{t})\right)$. Considering a point on the boundary surface, we find that scalar products $\mathbf{n}^{*} \cdot \mathbf{v}_{j}^{*}=\mathbf{n} \cdot \mathbf{v}_{j}, \mathbf{n}^{*} \cdot \mathbf{a}_{j}^{*}=\mathbf{n} \cdot \mathbf{a}_{j}$ and $R^{*}=R$ do not change, where * means image parameters. We can see that the tangential component of the sum of the original field and the image field on the grid surface is really zero. So for a task with delay, we can use the fields that are created by the images constructed in this way (Fig. 3).

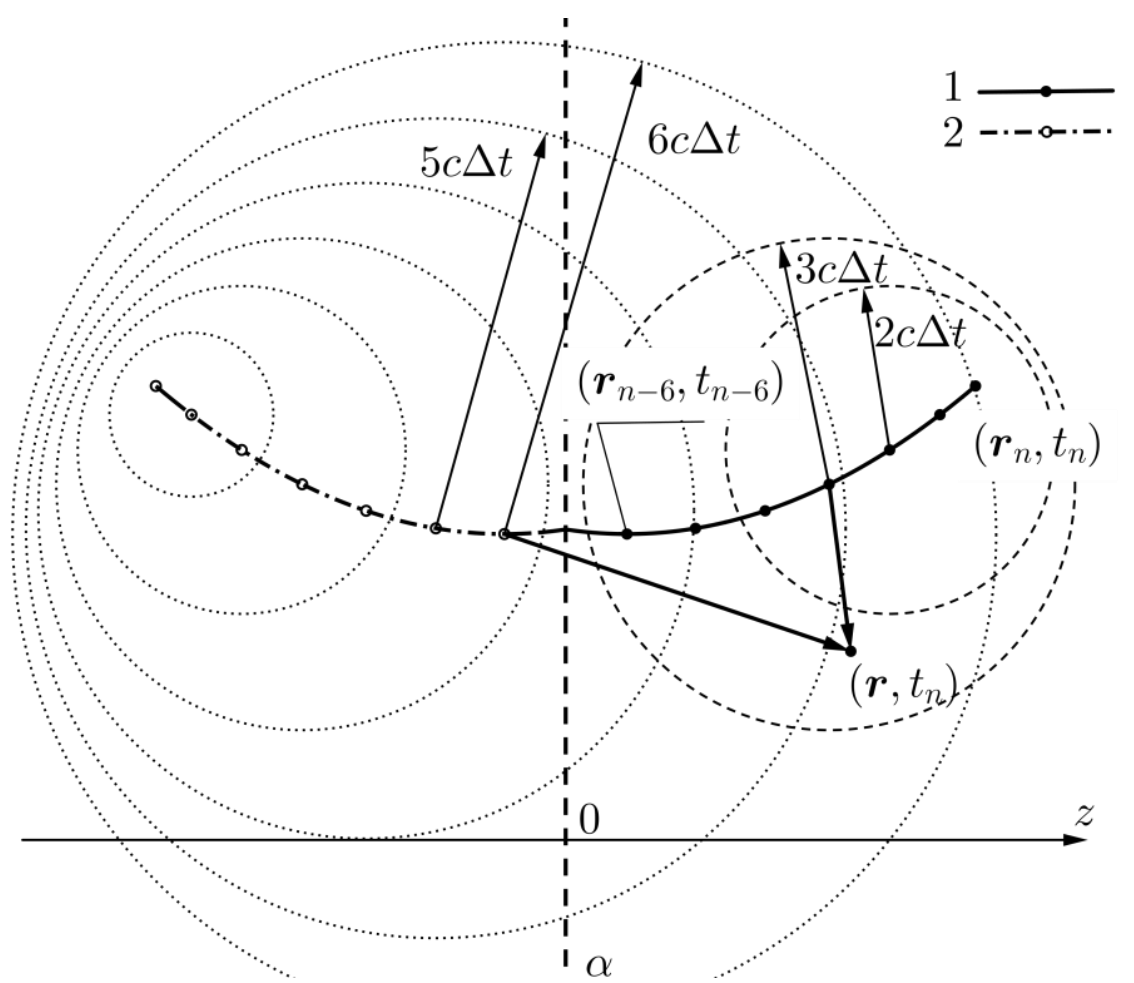

Fig. 3. The method of images for charged particles above the conducting plane $\alpha$. The field at this point is the sum of the fields of the original 1 and image 2, they are determined by the LienarWiechert formulas with the calculation of the time in the past by the bisection method. 


\section{Electron beam ejected into unlimited space}

The particle-particle calculation method does not allow taking into account complex boundary conditions. But we can consider the beam ejected into unlimited space that is equivalent to the interaction space with perfectly absorbing walls. And the image method allows us to compare two types of boundary conditions on the grid - perfectly absorbing and perfectly conducting.

We considered the cylindrical beam with the radius of $5.48 \mathrm{~mm}$. The time step in all calculations was $1 \mathrm{ps}$. The number of particles ejected per step into the interaction space was 30. Calculations were also performed with the smaller time step and the large number of ejected particles. A good agreement was found between the results by decreasing the step by a factor of 2 and 10 , and by increasing the number of particles by 2 .

Figure 4 shows a comparison of the phase space projection in coordinates $\left(v_{z}, z\right)$ for beams with perfectly absorbing boundary conditions and the perfectly conducting grid in the injection plane at different currents.
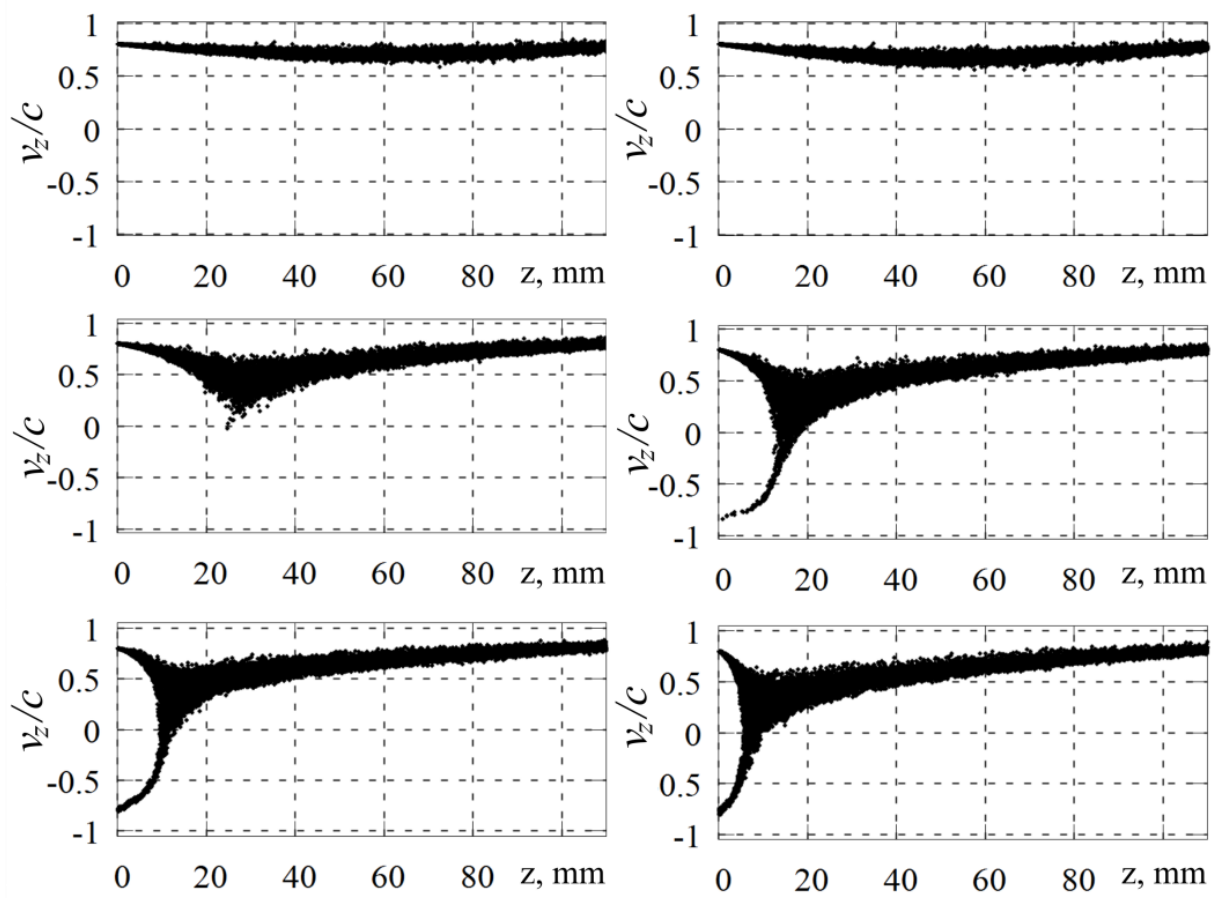

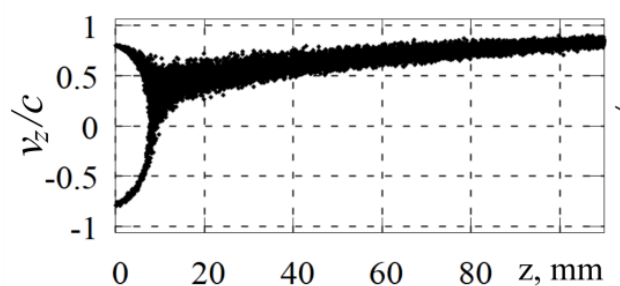

a)

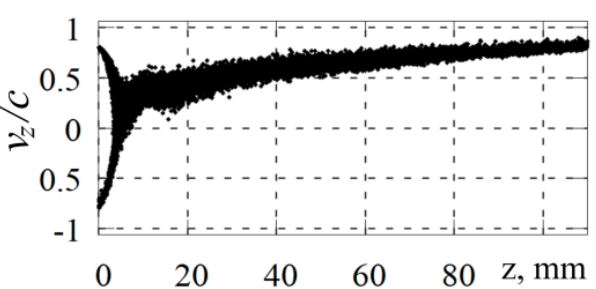

b)

Fig. 4. Projections of the phase space of the electron beam in the $\left(v_{z}, z\right)$ plane with increasing beam current a) without taking into account the image field $b$ ) with the images. System parameters: $v=0.8$ $\mathrm{c}, \mathrm{B}=1 \mathrm{~T}, \mathrm{r}=5.48 \mathrm{~mm}, \mathrm{~N}=30, \Delta \mathrm{t}=1 \mathrm{ps}, \mathrm{t}=600 \mathrm{ps}$. The current takes the values $\mathrm{I}=1 \mathrm{kA}, 2 \mathrm{kA}, 3$ $\mathrm{kA}, 5 \mathrm{kA}$ (from top to down). 
It can be seen from the figure that the time of formation of the virtual cathode and the plane of its formation strongly depend on the current and boundary conditions on the injector surface. In the case of the perfectly conducting surface we can observe the Schottky effect, the plane of the virtual cathode formation approaches the injector surface, and the time required for the formation of the virtual cathode decreases.

With increasing current, the space charge density increases. It means that interaction force increases with them. And it leads to the approach of the virtual cathode plane to the injection surface and reduces the time of its formation.

The maximum speed of electrons that move in the opposite direction is achieved in the injection plane and coincides with the initial electron velocity. The particle velocity after the virtual cathode gradually increases under the action of the force of the charged cloud, and for a small group of particles that has flown into the interaction space firstly can exceed the initial electron velocity.

Figure 5 shows phase portraits for the beams with different initial velocities and direct current. It is seen that with increasing speed, the time of formation of the virtual cathode increases, and the plane of the virtual cathode moves away from the injection plane. Taking into account for the conductive surface leads to the effect that the plane of formation of the virtual cathode approaches the injector plane, the formation time decreases.
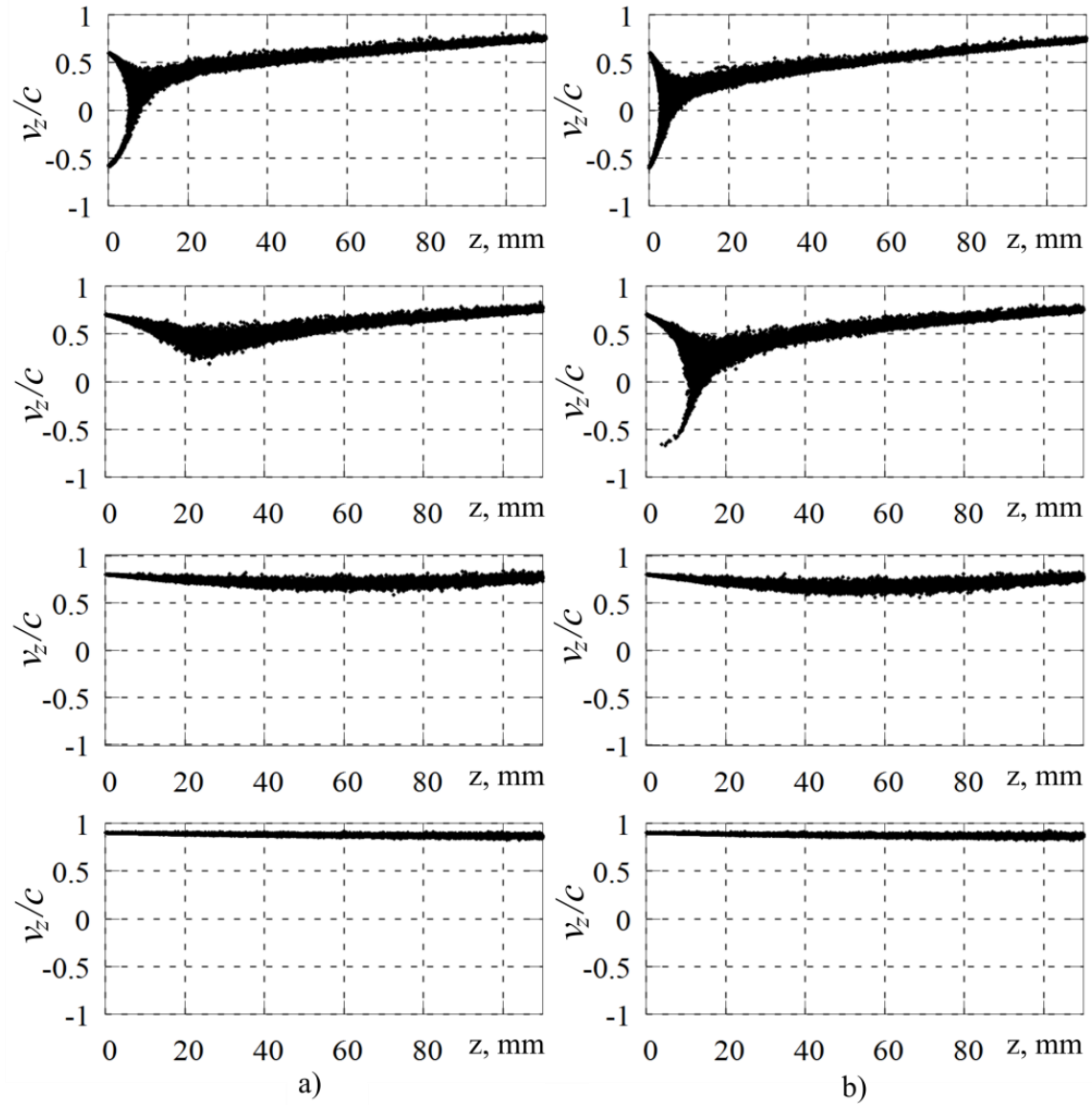

Fig. 5. Projections of the phase space of the electron beam in the $\left(v_{z}, z\right)$ plane with increasing beam velocity a) without taking into account the image field $b$ ) with the images. System parameters: $I=1$ $\mathrm{kA}, \mathrm{B}=1 \mathrm{~T}, \mathrm{r}=5.48 \mathrm{~mm}, \mathrm{~N}=30, \Delta \mathrm{t}=1 \mathrm{ps}, \mathrm{t}=600 \mathrm{ps}$. The velocity takes the values $\mathrm{v}=0.6 \mathrm{c}, 0.7 \mathrm{c}$, $0.8 \mathrm{c}, 0.9 \mathrm{c}$ (from top to bottom). 
The formation of the virtual cathode in the time interval from 0 to $600 \mathrm{ps}$ does not occur at high speeds. The space charge density is low, and the forces are small. For $0.9 \mathrm{c}$, the formation of the virtual cathode was also observed in these time intervals at high currents. Due to the nonlinear nature of the condition between the parameters of the current strength, velocity, and space charge density, it was necessary to consider one more case. Figure 6 shows phase portraits at the constant space charge density and various velocities. Increasing initial velocity we can see that the electron cloud start to move along the $\mathrm{z}$ axis. The beam has a large spread of speeds and ripples are noticeable in it. We can see all of the effects described above also.
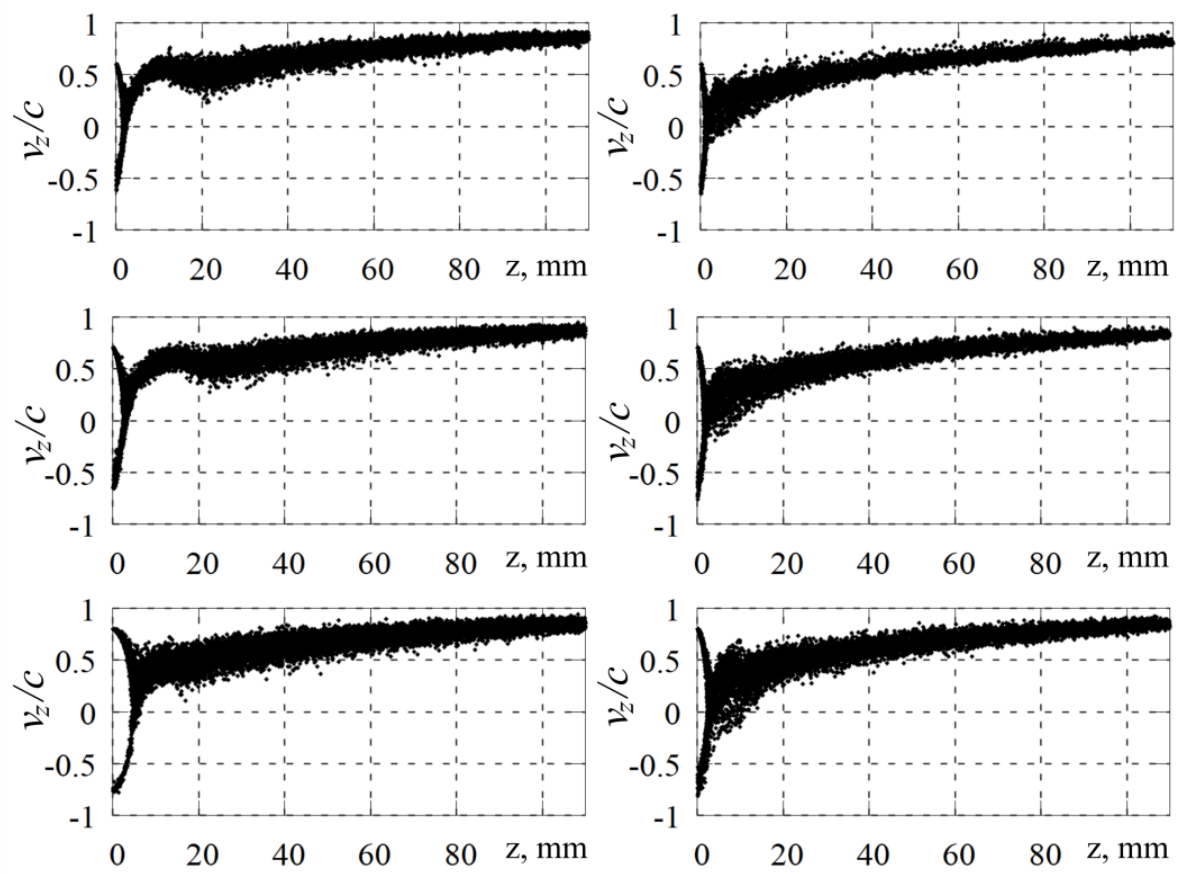

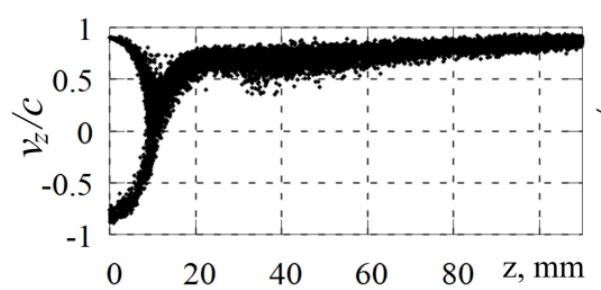

a)

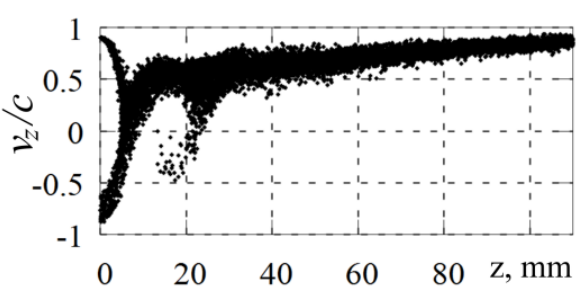

b)

Fig. 6. Projections of the phase space of the electron beam in the $\left(\mathrm{v}_{\mathrm{z}}, \mathrm{z}\right)$ plane with increasing beam velocity and constant space charge density a) without taking into account the image field $b$ ) with images. System parameters: $\rho=0.443 \mathrm{C} / \mathrm{m}^{3}$ (corresponds to $10 \mathrm{kA}$ at $0.8 \mathrm{c}$ ), $\mathrm{B}=1 \mathrm{~T}, \mathrm{r}=5.48 \mathrm{~mm}, \mathrm{~N}=$ $30, \Delta \mathrm{t}=1 \mathrm{ps}, \mathrm{t}=600 \mathrm{ps}$. The velocity takes the values $\mathrm{v}=0.6 \mathrm{c}, 0.7 \mathrm{c}, 0.8 \mathrm{c}, 0.9 \mathrm{c}$ (from top to bottom).

It follows from the above figures that the formation of the virtual cathode is a nonlinear effect in the parameters considered - current, velocity and space charge density. The plane of the virtual cathode formation, the time of its formation significantly depend on these characteristics, as well as on the type of boundary conditions in the injection plane.

The dependence of the time of the virtual cathode formation on velocity is shown in Figure 7. We understand the formation time as the time when 10 super-particles starts moving the opposite direction $\mathrm{z}$ axis. 


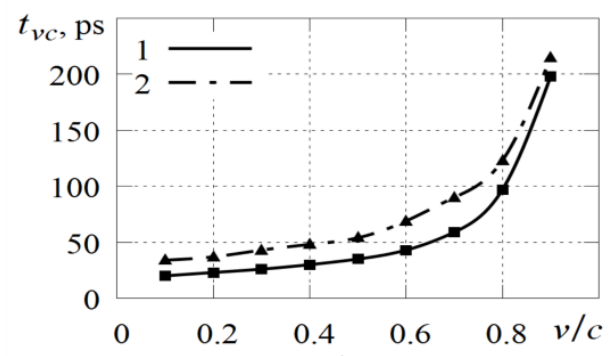

a)

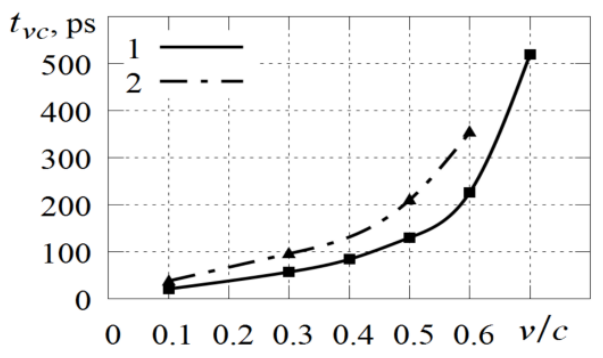

b)

Fig. 7. The virtual cathode formation time a) at constant space charge density b) at constant current of $1 \mathrm{kA}$ at speed of $0.8 \mathrm{c}$ and the initial beam geometry. 1 - with images, 2 - without images.

It follows from the figure that the influence of perfectly conducting boundary conditions leads to decreasing the formation time. Increasing the velocity at constant density the influence of the boundary conditions becomes less noticeable; on the contrary, increasing the velocity at constant current the space charge density decreases, and the virtual cathode does not form at speeds greater than a certain value in the considered time intervals.

\section{Conclusion}

The particle-particle simulation taking into account the delay effects does not allow studying complex devices with complex boundary conditions and materials, but it allows us to obtain numerical results for the nonlinear problem of the electron beam motion in external electromagnetic fields and to study the influence of the simplest boundary conditions in the form of perfectly conducting and perfectly absorbing planes. The specificity of these methods and the delay effect leads to some interesting effects - beam acceleration, the formation of the virtual cathode. It is usually assumed that the latter is formed when the current reaches a certain critical value. But studies carried out using particle-particle methods show that its formation in the system with the beam in unlimited space takes time and this time strongly depends on the type of boundary conditions on the injection surface. This phenomenon should be taken into account when designing devices with short relativistic electron pulses operating on the virtual cathode phenomenon.

\section{References}

1. Ju.N. Grigor'ev, V.A. Vshivkov, M.P. Fedoruk, Particle-in-cell numerical simulation, Izdatel'stvo SO RAN (2004)

2. T. Umeda, Y. Omura, T. Tominaga, H. Matsumoto, A new charge conservation method in electromagnetic particle-in-cell simulations, Computer Physics Communications, v. 156(1), pp. 73-85 (2003)

3. A.E. Dubinov, A.G. Petrik, S.A. Kurkin,N.S. Frolov, A.A. Koronovskii, A.E. Hramov, Virpertron: A novel approach for a virtual cathode oscillator design, Physics of Plasmas, 24, 073102 (2017)

4. S.V. Zaharchenko, A.G. Shein, Numerical modeling of extended relativistic electron flows, Izvestia VSTU, v. 32(6), pp 44-48 (2007)

5. D.G. Kovtun, A.G. Shein, A.G. Nasachjev, Modeling of the behavior of relativistic electron flow in crossed fields, Journal of Communications Technology and Electronics, v. 50(1), pp 108-111 (2005) 
6. D.G. Kovtun, P.D. Kravchenya, A.G. Shein, Influence of space interaction metal borders on the shape of relativistic electron beam moving in crossed fields Izvestia VSTU, v. 79(6), pp 30-36 (2011)

7. A.G. Shein, A.M. Bashkirev, Mechanism of pulsed generation mode when transporting a relativistic electron beam in a gas-filled medium, Infocommunications and Radio Technologies, v. 1(3), pp. 275-281, (In Russ.) (2018)

8. I.I. Magda, O.G. Melezhik, A.V. Pashchenko, S.S. Romanov, I.M. Shapoval, V.E. Novikov, Modification of the child-langmuir-boguslavsky law for the diode gap in the system with virtual cathode, Problems of Atomic Science and Technology. Series «Nuclear Physics Investigations», v. 80(4), pp 133-137 (2012) 